\title{
Modeling and Recognition of Landmark Image Collections Using Iconic Scene Graphs
}

\author{
Xiaowei Li, Changchang Wu, Christopher Zach, Svetlana Lazebnik, and \\ Jan-Michael Frahm \\ Dept. of Computer Science, University of North Carolina \\ Chapel Hill, NC 27599-3175 \\ \{xwli, ccwu, cmzach, lazebnik, jmf\}@cs.unc.edu
}

\begin{abstract}
This paper presents an approach for modeling landmark sites such as the Statue of Liberty based on large-scale contaminated image collections gathered from the Internet. Our system combines 2D appearance and 3D geometric constraints to efficiently extract scene summaries, build 3D models, and recognize instances of the landmark in new test images. We start by clustering images using low-dimensional global "gist" descriptors. Next, we perform geometric verification to retain only the clusters whose images share a common 3D structure. Each valid cluster is then represented by a single iconic view, and geometric relationships between iconic views are captured by an iconic scene graph. In addition to serving as a compact scene summary, this graph is used to guide structure from motion to efficiently produce 3D models of the different aspects of the landmark. The set of iconic images is also used for recognition, i.e., determining whether new test images contain the landmark. Results on three data sets consisting of tens of thousands of images demonstrate the potential of the proposed approach.
\end{abstract}

\section{Introduction}

The recent explosion in consumer digital photography and the phenomenal growth of photo-sharing websites such as Flickr.com have created a high demand for computer vision techniques for creating effective visual models from large-scale Internet-based image collections. Given a large database of images downloaded using a keyword search, the challenge is to identify all photos that represent the concept of interest and to build a coherent visual model of the concept despite heavy contamination by images with wrongly associated tags.

Recent literature contains a number of approaches that address the problem of visual learning from Internet image collections for general object categories (see, e.g., [1-3]). These approaches are well-adapted to deal with label uncertainty and make effective use of statistical appearance-based modeling, but lack strong geometric constraints that are needed for modeling categories with a common rigid 3D structure, such as famous tourist sites and landmarks. For modeling and visualization of landmarks from Internet images, structure-from-motion methods methods have been proposed $[4,5]$. These methods employ powerful 
geometric constraints and produce compelling 3D reconstructions, but are currently not very scalable and not well suited to take advantage of more than a small subset of a large and noisy community photo collection.

This paper presents a hybrid approach that combines the strengths of $2 \mathrm{D}$ recognition and 3D reconstruction for representing landmarks based on images downloaded from Flickr.com using keyword searches. Our system proceeds in an incremental fashion, initially applying 2D appearance-based constraints to loosely group images, and progressively refining these groups with geometric constraints to select iconic images for a sparse visual summary of the scene. These images and the pairwise geometric relationships between them define an iconic scene graph that captures all the salient aspects of the landmark. The iconic scene graph is then used for efficient reconstruction of a 3D skeleton model which can also be extended to many more relevant images to a comprehensive "collective representation" of the scene. The process of registering new test images to the model also allows us to answer the recognition question, namely, whether the landmark of interest is visible in a new test image. In addition, the iconic scene graph can be used to organize the image collection into a hierarchical browsing system. Because our method prunes many spurious images using fast $2 \mathrm{D}$ constraints and applies computationally demanding geometric constraints to just a small subset of "promising" images, it is scalable to large photo collections.

\section{Previous Work}

This paper offers a comprehensive solution to the problems of dataset collection, $3 \mathrm{D}$ reconstruction, scene summarization, browsing and recognition for landmark images. Below, we discuss related recent work in these areas.

The problem of dataset collection refers to the following: starting with the heavily contaminated output of an Internet image search query, extract a highprecision subset of images that are actually relevant to the query. Existing approaches to this problem [1-3] consider general visual categories not necessarily related by rigid 3D structure. They use statistical models to combine different kinds of 2D image features (texture, color, keypoints), as well as text and tags. However, 2D features alone do not provide strong enough constraints when applied to landmark images. Given the amount of clutter, viewpoint change, and lighting variation typically present in consumer snapshots, as well as the unreliability of user-supplied tags, it is difficult to answer the question of whether a landmark is actually present in a given picture without bringing in structurefrom-motion (SFM) constraints.

The Photo Tourism system of Snavely et al. [5] uses SFM constraints very effectively for modeling and visualization of landmarks. This system achieves high-quality reconstruction results with the help of exhaustive pairwise image matching and global bundle adjustment after inserting each new view. Unfortunately, this process becomes very computationally expensive for large data sets, and it is especially inefficient for heavily contaminated collections, most of whose images cannot be registered to each other. Accordingly, the input images used 
by Photo Tourism have either been acquired specifically for the task, or downloaded and pre-filtered by hand. When faced with a large and heterogeneous dataset, the best this method can do is use brute force to reduce it to a small subset that gives a good reconstruction. For example, for the Notre Dame results reported in [5] 2,635 images of Notre Dame were used initially, and out of these, 597 images were successfully registered after about two weeks of processing.

More recently, several researchers have developed SFM methods that exploit the redundancy in community photo collections to make reconstruction more efficient. In particular, many landmark image collections consist of a small number of "hot spots" from which photos are taken. Ni et al. [6] have proposed an out-of-core bundle adjustment approach that takes advantage of this by locally optimizing the "hot spots" and then connecting the local solutions into a global one. In this paper, we follow a similar strategy of computing separate $3 \mathrm{D}$ reconstructions on connected sub-components of the scene, thus avoiding the need for frequent large-scale bundle adjustment. Snavely et al. [7] find skeletal sets of images from the collection whose reconstruction provides a good approximation to a reconstruction involving all the images. Similarly, our method is based on finding a small subset of iconic images that capture all the important aspects of the scene. However, unlike $[6,7]$, we rely on 2D appearance similarity as a "proxy" or a rough approximation of the "true" multi-view relationship, and our goals are much broader: in addition to reconstruction, we are also interested in summarization, browsing, and recognition.

The problem of scene summarization for landmark image collections has been addressed by Simon et al. [8], who cluster images based on the output of exhaustive pairwise feature matching. While this solution is effective, it is perhaps too "strong" for the problem, as in many cases, a good subset of representative or "iconic" images can be obtained for a scene using much simpler 2D techniques [9]. This is the philosophy followed in our work: instead of treating scene summarization as a by-product of SFM, we treat it as a first step toward efficiently computing the scene structure.

Another problem relevant to our work is that of retrieval: given a query image, find all images containing the same landmark in some target database $[10,11]$. In this paper, we use retrieval techniques such as fast feature-based indexing and geometric verification with RANSAC to establish geometric relationships between different iconic images and to register a new test image to the iconics for the purpose of recognition.

\section{The Approach}

In this section, we present the components of our implemented system. Figure 1 gives a high-level summary of these components, and Figure 2 illustrates them with results on the Statue of Liberty dataset. 
1. Initial clustering (Section 3.1): Use "gist" descriptors to cluster the collection into groups roughly corresponding to similar viewpoints and scene conditions.

2. Geometric verification and iconic image selection (Section 3.2): The goal of this stage is to filter out the clusters whose images do not share a common $3 \mathrm{D}$ structure. This is done by pairwise epipolar geometry estimation among a few representative images selected from each cluster. The image that gathers the most inliers to the other representative images in its cluster is selected as the iconic image for that cluster.

3. Construction of iconic scene graph (Section 3.3): Perform pairwise epipolar geometry estimation among the iconic images and create an iconic scene graph by connecting pairs of iconics that are related by a fundamental matrix or a homography. Edges are weighted by the number of inliers to the transformation.

4. Tag-based filtering (Section 3.4): Use tag information to reject isolated nodes of the iconic scene graph that are semantically irrelevant to the landmark.

5. 3D reconstruction (Section 3.5): First, partition the iconic scene graph into several tightly connected components and compute structure from motion separately on each component. Within each component, use a maximum spanning tree to determine the order of registering images to the model. At the end, merge component models along cut edges.

6. Recognition (Section 4.2): Given a new test image, determine whether it contains an instance of the landmark. This can be done by efficiently registering the image to the iconics using appearance-based scores and geometric verification.

Fig. 1. Summary of the major steps of our system.

\subsection{Initial Clustering}

Our goal is to compute a representation of a landmark site by identifying a set of canonical or iconic views corresponding to dominant scene aspects. Recently, Simon et al. [8] have defined iconic views as representatives of dense clusters of similar viewpoints. To find these clusters, Simon et al. take as input a featureview matrix (a matrix that says which 3D features are present in which views) and define similarity of any two views in terms of the number of 3D features they have in common. By constrast, we adopt a perceptual or image-based perspective on iconic view selection: if there are many images in the dataset that share a very similar viewpoint in $3 \mathrm{D}$, then at least some of them will have a very similar image appearance in 2D, and can be matched efficiently using a low-dimensional global description of their pixel patterns.

The global descriptor we use is gist [12], which was found to be effective for grouping images by perceptual similarity [13]. We cluster the gist descriptors of all our input images using $k$-means with $k=1200$. Since at the next stage, we will select at most a single iconic image to represent each cluster, we initially want to produce an over-clustering to give us a large and somewhat redundant set of candidate iconics. In particular, we can expect images with very similar viewpoints to end up in different gist clusters because of clutter (i.e., people in front of the camera), differences in lighting, or camera zoom. This does not 


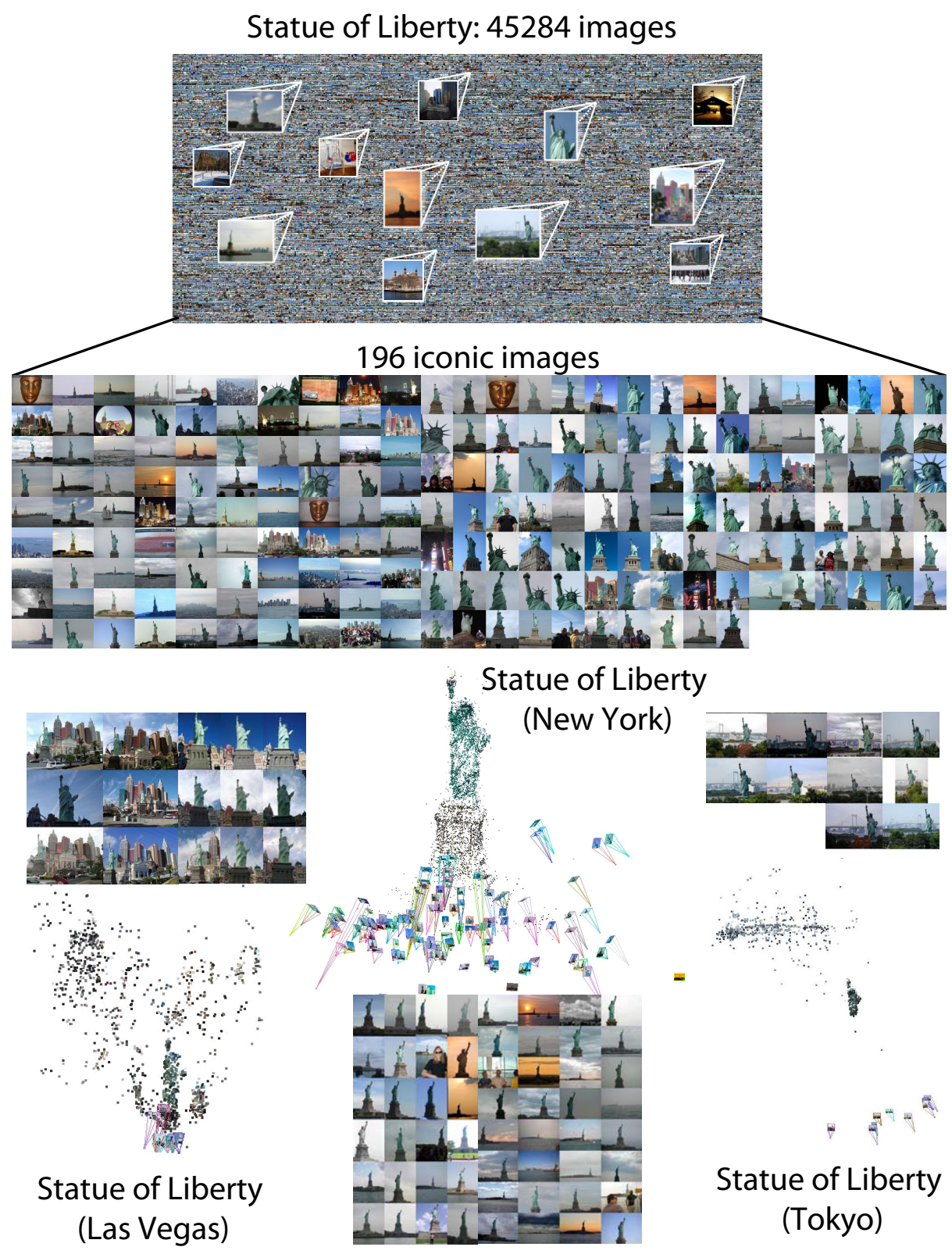

Fig. 2. A snapshot of the operation of our system for the Statue of Liberty. The initial dataset of 45284 images (of which about $40 \%$ are unrelated to the landmark) gets reduced to a set of 196 iconics by 2D appearance-based clustering followed by geometric verification of top cluster representatives. The iconics are nodes in a scene graph consisting of multiple connected components, each of which gives rise to a 3D reconstruction. The largest reconstructed component corresponds to the Statue of Liberty in New York, while two of the smaller ones correspond to copies of the statue in Tokyo and Las Vegas. These components are found completely automatically. A video of the models can also be found at http://www.cs.unc.edu/iconic-scene-graphs. 
cause a problem for our approach, because the graph construction step of Section 3.3 will be able to restore links between different clusters that have sufficient viewpoint similarity.

In our experiments, we have found that the largest gist clusters tend to be the cleanest ones. Therefore, for the initial stage, we use cluster size to produce a first, coarse ranking of images. As shown in the quantitative evaluation in Figure 6(a) (Stage 1), the first few gist clusters have a surprisingly high precision, though it deteriorates rapidly for subsequent clusters.

\subsection{Geometric Verification and Iconic Image Selection}

The next step is to perform verification of each gist cluster to confirm that its images share a common 3D structure. To do this efficiently, we select a small number of the most representative images from each cluster and attempt to estimate the two-view geometry of every pair. The representatives are given by $n$ images (in our current implementation, $n=8$ ) whose gist descriptors are closest to the cluster mean. Note that gist clusters that have fewer than $n$ images are rejected before this step.

For fitting a geometric transformation to these matches, we extract SIFT features [14] and use QDEGSAC [15], which is a robust procedure that returns an estimate for a fundamental matrix or a homography, depending on the scene structure. The image that gathers the largest total number of inliers to the other $n-1$ representatives from its cluster is declared the iconic image of that cluster. If any of the remaining representatives are not consistent with the iconic, we remove them and attempt to replace them with other images from the same cluster (within clusters, images are ranked in order of increasing distance from the center). If at the end of this process we are not able to find $n-1$ other consistent images, the cluster is rejected.

The inlier score of each iconic can be used as a new measure of the quality of each cluster. Precision/recall curves in Figure 6(a) (Stage 2) demonstrate that this ranking does a better job than gist alone in separating the relevant images from the irrelevant ones. However, there is an undesirable effect of a few geometrically consistent, but semantically irrelevant clusters getting very high scores at this stage, which hurts precision early on. Such clusters typically result from near-duplicate images coming from the same user's photo album. As described in the next section, we will be able to reject many such clusters using inter-cluster matching and filtering based on tags.

Ranking of clusters based on the top representatives does not penalize clusters that have a few geometrically consistent images, but are very low-precision otherwise. Once the iconic images for every cluster are selected, we can perform geometric verification of every remaining image by matching it to the iconic of its cluster and ranking it individually by the number of inliers it has with respect to its iconic. As shown in Figure 6(a) (Stage 3), this individual ranking improves precision considerably. 


\subsection{Construction of Iconic Scene Graph}

Next, we need to establish links between the iconic images selected in the previous step. Since we have hundreds of iconic images even following rejection of geometrically inconsistent clusters, exhaustive pairwise matching of all iconics is still rather inefficient. To match different iconic images, we need to account for larger viewpoint and appearance changes than in the initial clustering, so keypoint-based methods are more appropriate for this stage. We use the vocabulary tree method of Nister and Stewenius [16] as a fast indexing scheme to obtain promising candidates for pairwise geometric verification. We train a vocabulary tree with five levels and a branching factor of 10 using a set of thousands of frames taken from a video sequence of urban data, and populate it with SIFT features extracted from our iconics. We then use each iconic as a query image and perform geometric verification with top 20 other iconics returned by the vocabulary tree. Pairs of iconics that match with more than 18 inliers are then connected by an edge whose weight is given by the inlier score. This results in an undirected, weighted iconic scene graph whose nodes corresond to iconic views and edges correspond to two-view transformations (homographies or fundamental matrices) relating the iconics.

Next, we would like to identify a small number of strongly connected components in the iconic scene graph. This serves two purposes. The first is to group together iconics that are close in terms of viewpoint but did not initially fall into the same gist cluster. The second is to obtain smaller subsets of images on which structure from motion can be performed more efficiently. To partition the graph, we use normalized cuts [17], which requires us to specify as input the desired number of components. This parameter choice is not critical, since any oversegmentation of the graph will be addressed by the component merging step discussed in the next section. We have found that specifying a target number of 20 to 30 components produces acceptable results for all our datasets.

Every (disjoint) component typically represents a distinctive aspect of the landmark, and we can select a single representative iconic for each component (i.e., the iconic with the highest sum of edge weights) to form a compact scene summary. Moreover, the components of the iconic scene graph and the iconic clusters induce a hierarchical structure on the dataset that can be used for browsing, as shown in Figure 3.

\subsection{Tag-Based Filtering}

The iconic scene graph tends to have many isolated nodes, corresponding to iconic views for which we could not find a geometric relationship with any other view. These nodes are excluded from the graph partitioning process described above. They may either be aspects of the scene that are significantly different from others, e.g., the interior of the Notre Dame cathedral in Paris, or geometrically consistent, but semantically irrelevant clusters, e.g., pictures of a Notre Dame cathedral in a different city. Since constraints on appearance and geometry are not sufficient to establish the relationship of such clusters to the scene, to 


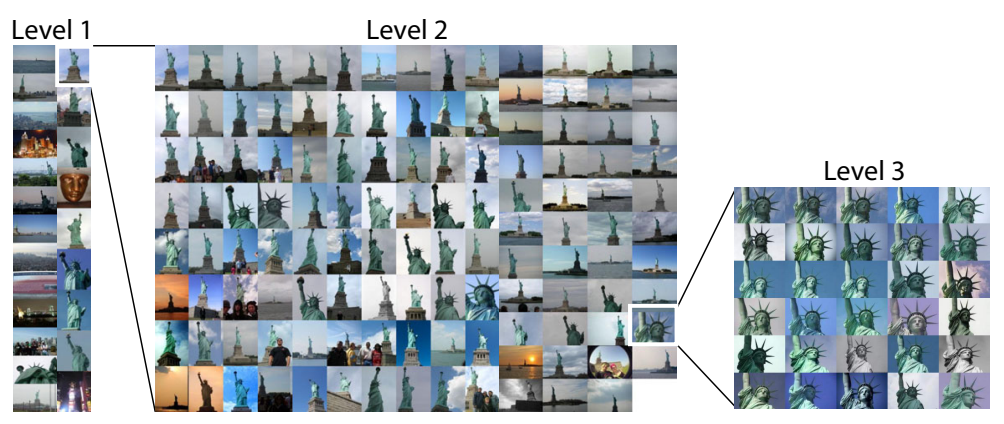

Fig. 3. Hierarchical organization of the dataset for browsing. Level 1: components of the iconic scene graph. Level 2: Each component can be expanded to show all the iconic images associated with it. Level 3: each iconic can be expanded to show the images associated with its gist cluster. Our three datasets may be browsed online at http://www.cs.unc.edu/iconic-scene-graphs.

refine our dataset further we need to use additional information, in particular, the tags associated with the images on Flickr.

Even though Flickr tags in general tend to be quite unreliable, we have observed that among the isolated clusters that have already been pre-filtered by appearance and geometry constraints, there are quite a few whose tags are clearly unrelated to the landmark. This suggests that, provided we have a good idea of the distribution of relevant tags, a very simple score should be sufficient to identify the "bad" clusters. Fortunately, during the previous modeling stages, we have already verified hundreds of images without resorting to tags, so we can now use these images to acquire the desired distribution. In the implementation, we take iconic images that have at least two edges in the scene graph (empirically, these are almost certain to contain the landmark), and use them to create a "master list" of relevant tags. To have a more complete list, we also incorporate tags from the top cluster images registered to these iconics. The tags in the list are ranked in decreasing order of frequency, and isolated iconic images are scored based on the median rank of their tags (tags that don't occur in the master list at all are assigned an arbitrary high number). Clusters with "obviously" unrelated tags get a high median rank and can be removed to increase precision, as shown by the "Stage 4" curves in Figure 6(a).

\subsection{D Reconstruction}

As a first step, the 3D structure for every component produced by normalized cuts is computed separately. Starting with a good initial image pair, we incrementally add more views to the reconstruction by perspective pose estimation. There are two criteria for selecting a good initial pair. First, in order to operate in metric instead of projective space, the views in question require reasonable estimates for the focal lengths, which can be obtained from EXIF data. When 


\begin{tabular}{l||rrr|rr||rr|rr}
\multicolumn{1}{c}{} & \multicolumn{3}{c}{ Modeling } & \multicolumn{3}{c}{ Testing } & \multicolumn{2}{c}{ All 3D models } & \multicolumn{2}{c}{ Largest 3D model } \\
Dataset & Unlabeled & Pos. & Neg. & Pos. & Neg. & \#Models & \#Views & \#Views & \#Pts \\
\hline Notre Dame & 9760 & 545 & 535 & 541 & 503 & 8 & 580 & 337 & 30802 \\
Statue of Liberty & 42983 & 1369 & 932 & 646 & 446 & 6 & 1068 & 871 & 18675 \\
San Marco & 38332 & 2094 & 3131 & 379 & 715 & 4 & 1213 & 749 & 39307
\end{tabular}

Table 1. Summary statistics of our datasets and 3D models. The first five columns list dataset sizes and numbers of labeled images. The next two columns give details of our computed 3D models: the number of distinct models after merging and the total number of registered views (these include both iconic and non-iconic images). The last two columns refer to just the single largest 3D model. They list the number of registered views and the number of $3 \mathrm{D}$ points visible in at least three views.

EXIF data is not available, we transfer the focal length estimate from similar views in the same cluster. Second, the number of inlier correspondences should be as large as possible, taking into account the 3D point triangulation certainty (i.e. the shape of the covariance matrices) [18]. Once an initial pair of images is found, their relative pose is determined by the five-point method [19]. The remaining views of the current component are added using perspective pose estimation from 2D-3D correspondences. The order of insertion is determined from the edges of the underlying maximum spanning tree computed for the weighted graph component. Hence, views having more correspondences with the current reconstruction are added first. The resulting 3D structure and the camera parameters are optimized by non-linear sparse bundle adjustment [20].

The above reconstruction process applied to individual graph components produces small individual models representing single aspects of the landmark. A robust estimation of the $3 \mathrm{D}$ similarity transform is used to align and merge suitable components and their respective reconstructions. This merging process restores the connectivity of the original scene graph, and results in a single "skeleton" 3D model for each original connected component. The last step is to augment these models by incorporating additional non-iconic images from clusters. Each image we consider for adding has already been successfully registered with the iconic of its cluster, as described in Section 3.2. Since the features from the iconic have already been incorporated into the skeleton 3D model, the registration between the image and the iconic gives us a number of $2 \mathrm{D} / 3 \mathrm{D}$ matches for that image. To obtain additional 2D/3D matches, we attempt to register this new image to two additional iconics that are connected to its original iconic by the highest-weight edges. All these matches are then used to estimate the pose of the new image. At the end, bundle adjustment is applied to refine the model.

\section{Experimental Results}

\subsection{Data Collection and Model Construction}

We have tested our system on three datasets: the Notre Dame cathedral in Paris, the Statue of Liberty in New York, and Piazza San Marco in Venice. The datasets were automatically downloaded from Flickr.com using keyword 


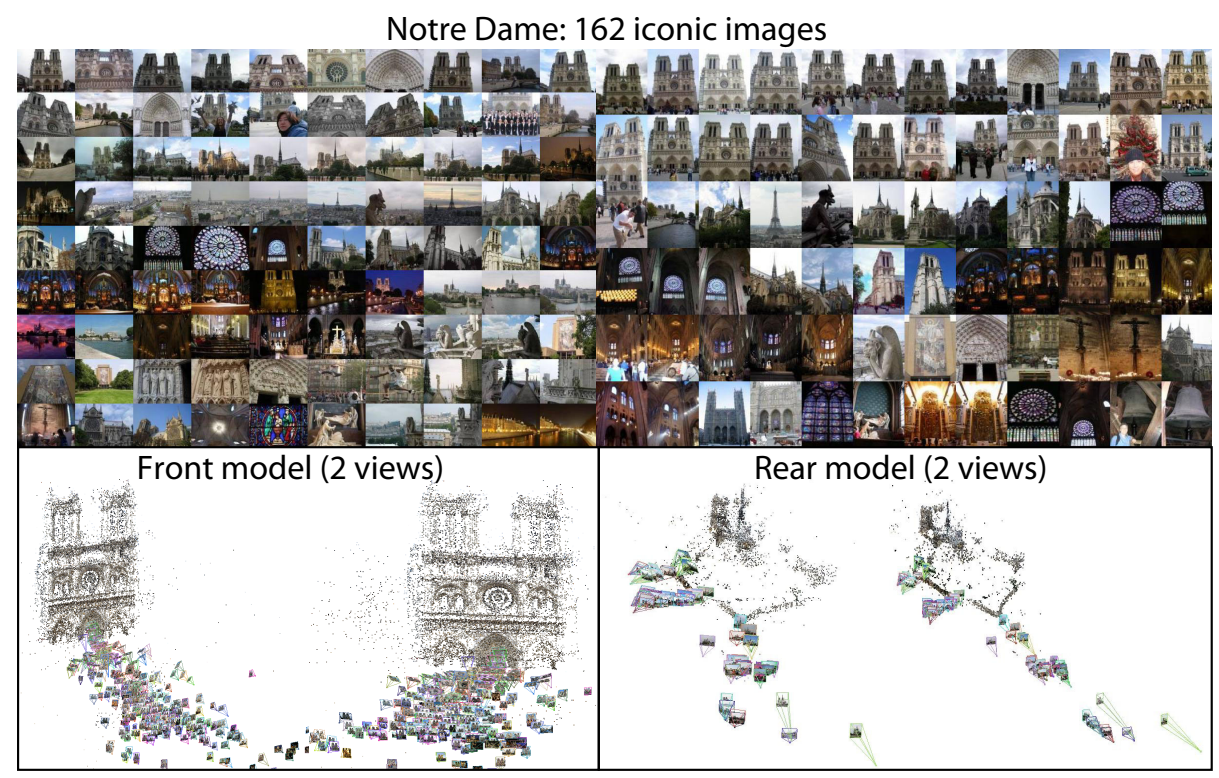

Fig. 4. Notre Dame results. Top: iconic images. Note that there are a few spurious iconics corresponding to Notre Dame cathedrals in Indiana and Montreal (these were not removed by the tag filtering step), as well as the Eiffel Tower seen from the top of Notre Dame. Bottom: two of the reconstructed scene components, corresponding to the front and back of the cathedral.

searches. We randomly split each dataset into a "modeling" part, and a much smaller independent "testing" part. Because the modeling datasets contain tens of thousands of images, we have chosen to label only a small randomly selected fraction of them. These ground-truth labels are needed only to measure recall and precision for the different stages of refinement, since our modeling approach is completely unsupervised. The smaller test sets are completely labeled. Our labeling is very basic, merely recording whether the landmark is present in the image or not, without evaluating the "quality" or "typicality" of a given view. For example, interior views of Notre Dame are labeled as positive, even though they are relatively few in number and cannot be registered to the exterior views. Table 1 gives a breakdown of the numbers of labeled and unlabeled images in our datasets. The proportions of negative images ( $40 \%$ to $60 \%$ ) give a good idea of the initial amount of contamination.

Figure 6(a) shows recall/precision curves for the modeling process on the three datasets. We can see that the four refinement stages (gist clustering, geometric verification of clusters, verification of individual images w.r.t. their cluster centers, and tag-based rejection) progressively increase the precision of the images selected as part of the model, even though recall decreases following the rejection decisions made after every stage. 


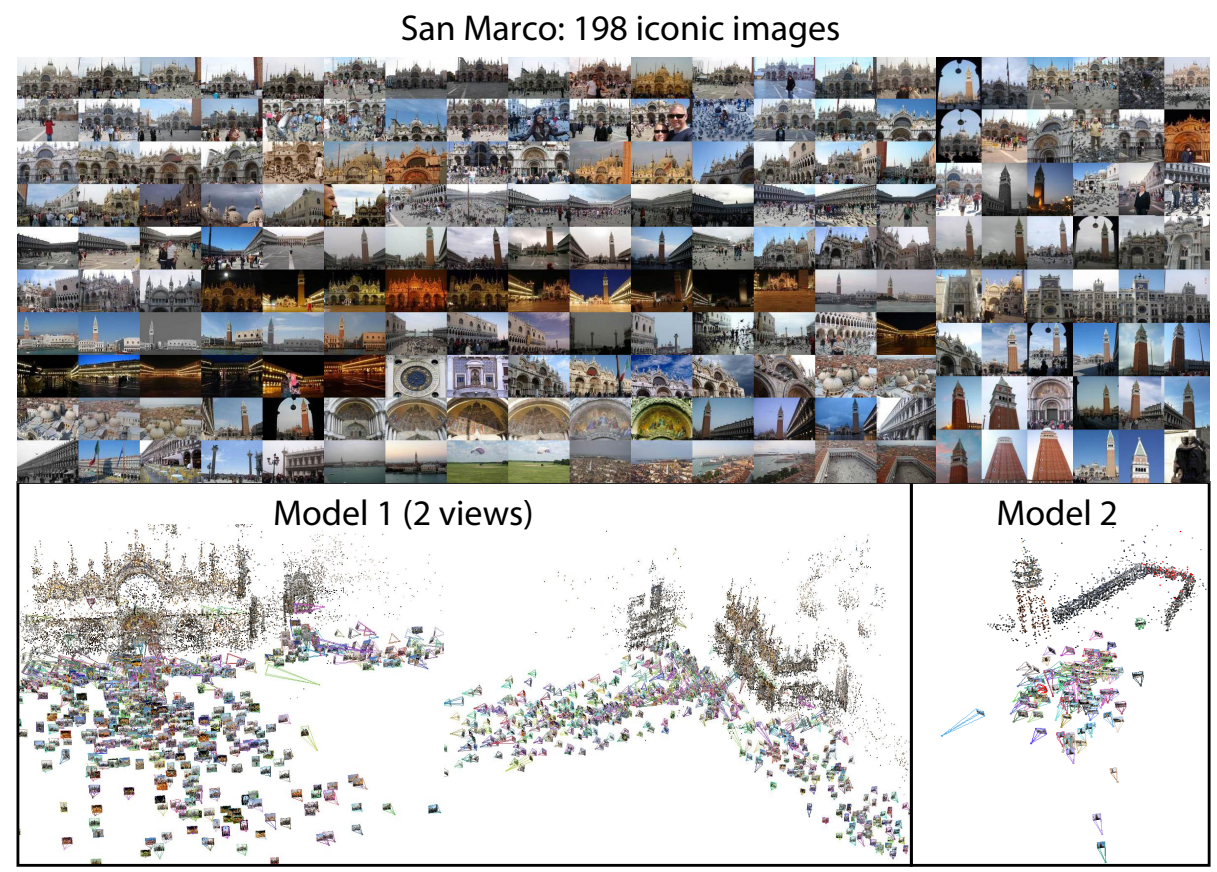

Fig. 5. San Marco results. Top: iconic images. Bottom: two of the reconstructed scene components, corresponding to the front and the back of the square.

Figures 2, 4 and 5 show the reconstructed 3D models for our three datasets (see http://www.cs.unc.edu/iconic-scene-graphs for videos of the models). As described in Section 3.5, reconstruction is first performed on separate components of the iconic scene graph, followed by merging of models with sufficiently overlapping scene components. Successful merging requires images that link the component models. For San Marco, the merging of the models corresponding to the front and back of the square was not successful because the iconic images did not provide a sufficient coverage of the middle region. For a similar reason, it was not possible to merge the front and the back of Notre Dame. To an extent, this merging problem is endemic to community photo collections, as people tend to take snapshots of famous landmarks from a small number of particularly characteristic or accessible "hot spots," while the areas in between remain sparsely covered. Our clustering approach may in some cases exacerbate this problem by discarding the less common images that fail to produce sufficiently large clusters. Despite the difficulty of merging, our models successfully incorporate a significant number of images, as shown in Table 1. While our models currently do not exceed in size those produced by the Photo Tourism system [5], we are able to process an order of magnitude more images with just a fraction 
of the computational power, i.e., hours on a single commodity PC, instead of weeks on a high-performance cluster.

\subsection{Testing and Recognition}

Given a new image that was not in our initial collection, we want to find out whether it contains the landmark of interest. A straightforward of doing this is by retrieving the iconic image that gets the highest matching score with the test image (according to a given retrieval scheme) and making the yes/no decision by setting a threshold on the retrieval score. We can evaluate performance quantitatively by plotting a recall/precision curve of the test images ordered from highest to lowest score. Figure 6 (b) and (c) shows the results for several retrieval strategies. The simplest strategy is to compare the test image to the iconics using either gist descriptors (in which case the score would be inversely proportional to the distance) or a bag-of-features representation using the vocabulary tree (which returns a tf/idf score [16]). For improved performance, we can take top $k$ "candidate" iconics retrieved with either gist or vocabulary tree, and perform geometric verification with each candidate as described in Section 3.2. In this case, the score for each candidate is the number of inliers to a twoview transformation (homography or fundamental matrix) between it and the test image, and only the top candidate is retained.

Interestingly, for the Statue of Liberty, the performance of the vocabulary tree without geometric verification is almost disastrous. This is due to the relative lack of texture in many Statue of Liberty images, which gives too few local features for bag-of-words matching to work reliably. But in most other cases, gist and vocabulary tree have comparable performance. Not surprisingly, for both kinds of image description, geometric verification significantly improves accuracy, as does retrieving more candidates for verification. For comparison, we also include a recall/precision curve for scoring test images based on their tag relevance (see Section 3.4). By itself, this scoring scheme is quite unreliable.

\section{Discussion}

We have presented a hybrid approach combining 2D appearance and 3D geometry to efficiently model and recognize complex real-world scenes captured by thousands of amateur photos. To our knowledge, our system is the first integrated solution to the problems of dataset collection, scene summarization, browsing, 3D reconstruction, and recognition for landmark images. At the heart of our approach is the iconic scene graph, which captures the major aspects of the landmark and the geometric connections between them. The structure of this graph is used, among other things, to create a three-level browsing hierarchy and to enable scalable computation of structure from motion.

In the future, one of our main goals is to improve the recall of our modeling process. This can be done by making use of fast and accurate retrieval techniques such as $[10,11]$ to re-incorporate images that were discarded during the iconic 
(a)
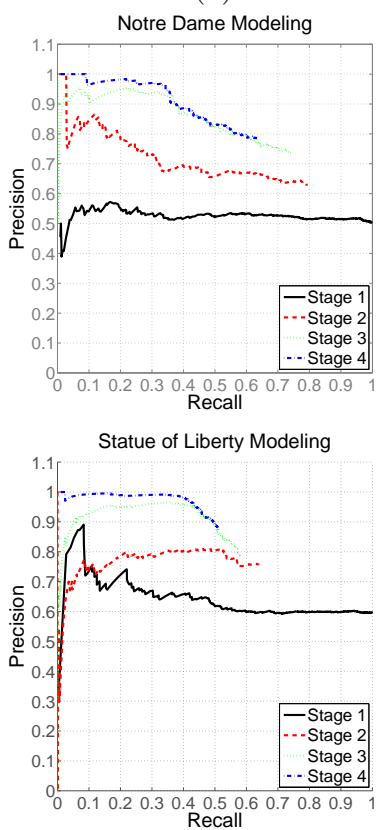

San Marco Modeling

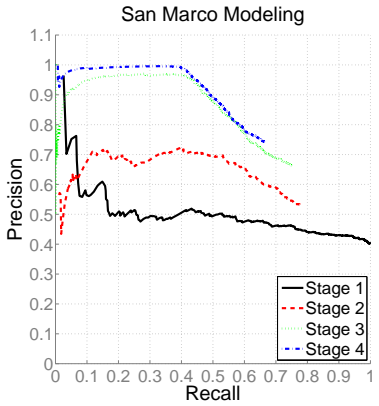

(b)

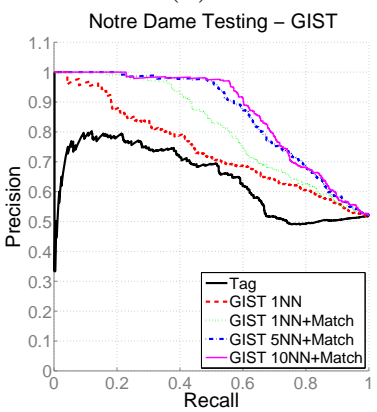

Statue of Liberty Testing - GIST

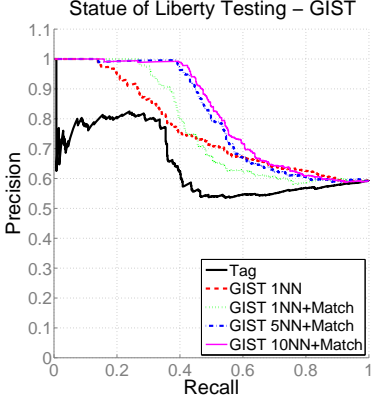

San Marco Testing - GIST

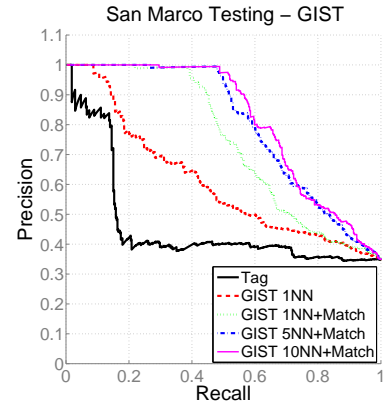

(c)

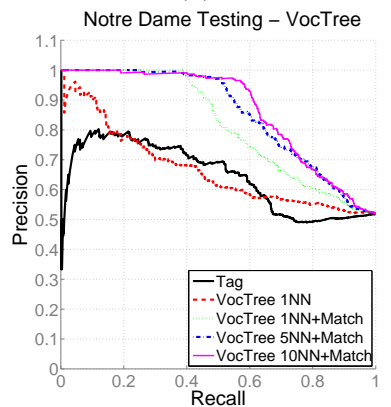

Statue of Liberty Testing - VocTree

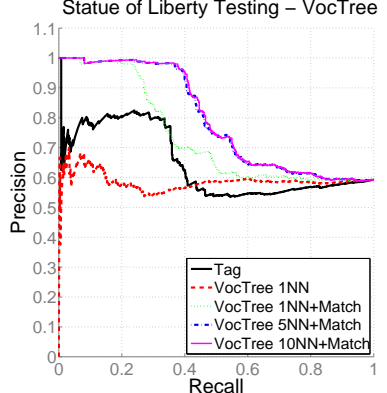

San Marco Testing - VocTree

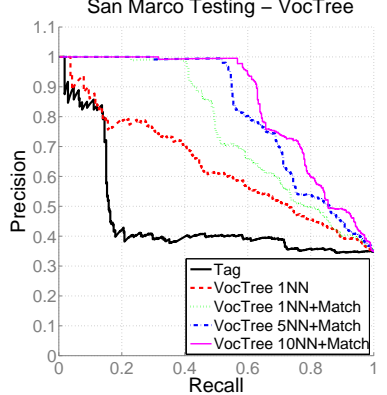

Fig. 6. Recall/precision curves for (a) modeling; (b) testing with the gist descriptors; and (c) testing with the vocabulary tree. For modeling, the four stages are as follows. Stage 1: Clustering using gist and ranking each image by the size of its gist cluster (Section 3.1). Stage 2: Geometric verification of iconics and ranking each image by the inlier number of its iconic (Section 3.2). The recall is lower because inconsistent clusters are rejected. Stage 3: Registering each image to its iconic and ranking the image by the number of inliers of the two-view transformation to the iconic. Unlike in the first two stages, images are no longer arranged by cluster, but ranked individually by this score. The recall is lower because images with not enough inliers to estimate a two-view transformation are rejected. Stage 4: Tag information is used to retain only the top 30 isolated clusters (Section 3.4). The score is the same as in stage 3 , except that images belonging to the rejected clusters are removed. Note the increase in precision in the first few retrieved images. For testing, the different retrieval strategies are as follows. GIST 1NN (resp. VocTree 1NN): retrieval of the single nearest iconic using the gist descriptor (resp. vocabulary tree); GIST kNN+Match (resp. VocTree kNN+Match): retrieval of $k$ nearest exemplars using gist (resp. vocabulary tree) followed by geometric verification; Tag: tag-based ranking (see Section 3.4). 
image selection stage. A similar strategy could also be helpful for discovering "missing links" for merging 3D models of different components. In addition, we plan to create 3D models that incorporate a much larger number of images. This will require a memory-efficient streaming approach for registering new images, as well as out-of-core bundle adjustment using iconic scene graph components.

\section{References}

1. Fergus, R., Perona, P., Zisserman, A.: A visual category filter for Google images. In: ECCV. (2004)

2. Berg, T., Forsyth, D.: Animals on the web. In: CVPR. (2006)

3. Schroff, F., Criminisi, A., Zisserman, A.: Harvesting image databases from the web. In: ICCV. (2007)

4. Goesele, M., Snavely, N., Curless, B., Hoppe, H., Seitz, S.M.: Multi-view stereo for community photo collections. In: ICCV. (2007)

5. Snavely, N., Seitz, S.M., Szeliski, R.: Photo tourism: Exploring photo collections in 3d. In: SIGGRAPH. (2006) 835-846

6. Ni, K., Steedly, D., Dellaert, F.: Out-of-core bundle adjustment for large-scale 3d reconstruction. In: ICCV. (2007)

7. Snavely, N., Seitz, S.M., Szeliski, R.: Skeletal sets for efficient structure from motion. In: CVPR. (2008)

8. Simon, I., Snavely, N., Seitz, S.M.: Scene summarization for online image collections. In: ICCV. (2007)

9. Berg, T.L., Forsyth, D.: Automatic ranking of iconic images. Technical report, University of California, Berkeley (2007)

10. Chum, O., Philbin, J., Sivic, J., Isard, M., Zisserman, A.: Total recall: Automatic query expansion with a generative feature model for object retrieval. In: ICCV. (2007)

11. Philbin, J., Chum, O., Isard, M., Sivic, J., Zisserman, A.: Lost in quantization: Improving particular object retrieval in large scale image databases. In: CVPR. (2008)

12. Oliva, A., Torralba, A.: Modeling the shape of the scene: a holistic representation of the spatial envelope. IJCV 42 (2001) 145-175

13. Hays, J., Efros, A.A.: Scene completion using millions of photographs. In: SIGGRAPH. (2007)

14. Lowe, D.: Distinctive image features from scale-invariant keypoints. IJCV 60 (2004) 91-110

15. Frahm, J.M., Pollefeys, M.: RANSAC for (quasi-)degenerate data (QDEGSAC). In: CVPR. Volume 1. (2006) 453-460

16. Nister, D., Stewenius, H.: Scalable recognition with a vocabulary tree. In: CVPR. (2006)

17. Shi, J., Malik, J.: Normalized cuts and image segmentation. PAMI 22 (2000) 888-905

18. Beder, C., Steffen, R.: Determining an initial image pair for fixing the scale of a 3d reconstruction from an image sequence. In: Proc. DAGM. (2006) 657-666

19. Nistér, D.: An efficient solution to the five-point relative pose problem. PAMI 26 (2004) 756-770

20. Lourakis, M., Argyros, A.: The design and implementation of a generic sparse bundle adjustment software package based on the Levenberg-Marquardt algorithm. Technical Report 340, Institute of Computer Science - FORTH (2004) 